\title{
Controlling conducting channels of single-walled carbon nanotube array with atomic force microscopy
}

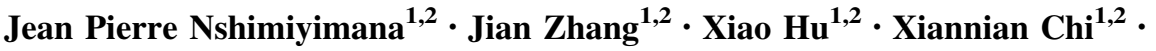 \\ Pei $\mathrm{Wu}^{1,2} \cdot$ Siyu Liu ${ }^{1} \cdot$ Zongzhi Zhang ${ }^{1} \cdot$ Weiguo Chu ${ }^{1} \cdot$ Lianfeng Sun $^{1}$
}

Received: 14 August 2017/ Accepted: 4 October 2017/Published online: 17 October 2017

(C) The Author(s) 2017. This article is an open access publication

\begin{abstract}
In this paper, we report a technique of controlling the number of single-walled carbon nanotubes (SWNTs) between two electrodes. The SWNT devices consist of an array of SWNTs between two silver/palladium electrodes, which are fabricated with electron beam lithography and silver. Using the tip-sample interaction in contact mode of atomic force microscopy (AFM), the number of individual SWNTs can be controllably decreased. The current-voltage measurements indicate that
\end{abstract}

Lianfeng Sun

slf@nanoctr.cn

Jean Pierre Nshimiyimana

peternshi@nanoctr.cn

Jian Zhang

zhangjian@nanoctr.cn

Xiao $\mathrm{Hu}$

hux@nanoctr.cn

Xiannian Chi

chixn@nanoctr.cn

Pei Wu

wup@nanoctr.cn

Siyu Liu

liusy@nanoctr.cn

Zongzhi Zhang

zhangzz@nanoctr.cn

Weiguo Chu

wgchu@ nanoctr.cn

1 CAS Key Laboratory of Nanosystem and Hierarchical Fabrication, CAS Center for Excellence in Nanoscience, National Center for Nanoscience and Technology, Beijing 100190, China

2 University of Chinese Academy of Sciences, Beijing 100049, China the resistance increases when the conducting channels of SWNTs are reduced. This simple and controllable approach could be useful for the assembly of high performance devices with a desired number of channels for future electronic investigations.

Keywords Single-walled carbon nanotube - Atomic force microscopy $\cdot$ Manipulation $\cdot$ Conducting channels

\section{Introduction}

Ever since their discovery, carbon nanotubes (CNTs) have attracted tremendous attention due to their unique structure and outstanding mechanical and electronic properties (Baughman et al. 2002; Fischer and Johnson 1999; Louie 2001; Salvetat et al. 1999). Single-walled carbon nanotubes (SWNTs) consist of a single graphene sheet wrapped up into a cylindrical tube. The band structure of SWNTs depends on their diameter and chiral angle and hence, they can be either semiconducting or metallic. Owing to these unusual electronic characteristics, SWNTs have been considered as an ideal candidate in a variety of nanoscale devices, such as transistors (Ionescu and Riel 2011; Li et al. 2014), sensors (Karimov et al. 2015; Kauffman and Star 2008), etc. However, the fabrication of SWNT-based devices still presents a challenging task, mostly owing to the difficulties in controlling their density, diameter and placement (De Volder et al. 2013). Consequently, to build SWNT-based devices with preferred structure and electronic properties, it is of great importance to know how to manipulate the nanotubes individually and place them at desired positions. Atomic force microscopy (AFM) has been a widely used tool to perform various kinds of CNT manipulation. Both contact mode and non-contact mode 
could be used to translate, bend, roll, split or cut the nanotubes (Hyon et al. 2005; Kim et al. 2003; Kumar et al. 2012; Postma et al. 2000; Ziyong et al. 2003), either by applying the tip bias (Hyon et al. 2005; Kim et al. 2003; Park et al. 2002) or by exerting mechanical forces (Hertel et al. 1998). Devices based on SWNTs were also fabricated through AFM manipulation such as single-electron transistors (Postma et al. 2001), field-effect transistors (Avouris et al. 1999), diodes (Jiao et al. 2008), and so on.

For the fabrication of SWNT array devices, several SWNTs need to be placed between a pair of electrodes as source and drain (Zhang 2013). Although these devices produce high output currents (Seidel et al. 2004), their overall performance may be hampered by the random alignment of SWNT channels. Here, we present a technique of controlling the number of conducting channels of SWNTs between a pair of electrodes. Using the tip of AFM in contact mode, an array of SWNTs could be controllably decreased into a well-defined number of channels between the electrodes. Efficient parameters are established for the manipulation of the nanotubes. It was found that with no tip bias, the SWNT bundles are disentangled into fluffy SWNTs and could only be cut and swept away when the tip voltage is applied. The change in electrical resistance with the decrease of the SWNT conducting channels is also discussed. This technique provides possibilities for the assembly of high performance devices with controllable electronic properties.

\section{Experimental}

The SWNT samples used in this work were prepared as follows. A silicon wafer coated with 800 -nm-thick $\mathrm{SiO}_{2}$ layer was used as substrate. Standard electron beam lithography (EBL) and lift-off process were used to fabricate an electrode pattern on top of which palladium electrodes were evaporated $(5 \mathrm{~nm} \mathrm{Cr} / 100 \mathrm{~nm} \mathrm{Pd})$. A layer of PMMA was spin coated on the substrate and an aligned pattern was made on the electrode area by EBL. A 400-nmthick silver (Ag) film was then evaporated on the substrate. SWNTs were grown on the silver surface using floatingcatalytic chemical vapour deposition (Zhao et al. 2008) in a mixture of $1000 \mathrm{sccm} \mathrm{Ar}$ and $10 \mathrm{sccm} \mathrm{CH}_{4}$ at $1000{ }^{\circ} \mathrm{C}$. Finally, following a lift-off process to remove unwanted $\mathrm{Ag}$ particles and SWNTs on PMMA, the sample was subjected to a thermal annealing treatment at $960{ }^{\circ} \mathrm{C}$. At this stage, the silver film melted into liquid and the randomly distributed SWNTs were pulled into a straightened array between the palladium electrodes.

The as-fabricated SWNT array samples were consequently manipulated using atomic force microscopy. The morphology and electrical characteristics of the samples were investigated by a field emission scanning electron microscope (SEM, Hitachi S-4800) and B1500 Agilent semiconductor characterization system, respectively. The AFM experiments were carried out at room temperature using a commercial multimode scanning probe microscope from Veeco instruments. A Pt/Ir-coated conductive cantilever, with a spring constant of $2.8 \mathrm{~N} / \mathrm{m}$ and resonant frequency of about $75 \mathrm{kHz}$, was used.

\section{Results and discussions}

In Fig. 1a, the SEM image of the fabricated SWNT array devices is shown. A set of five palladium electrodes labeled $1,2,3,4$ and 5 were fabricated on the substrate. The designed spacing between two electrodes (herein referred to as $1-2,2-3,3-4$ and $4-5$ ) was $1,2,3$ and $4 \mu \mathrm{m}$, respectively. The SEM result shows that the alignment of SWNTs is dependent on the electrode spacing, with the smallest spacing having the best alignment. Figure $1 b$ shows the typical room-temperature source-drain current $\left(I_{\mathrm{SD}}\right)$ and source-drain voltage $\left(V_{\mathrm{SD}}\right)$ characteristics of the
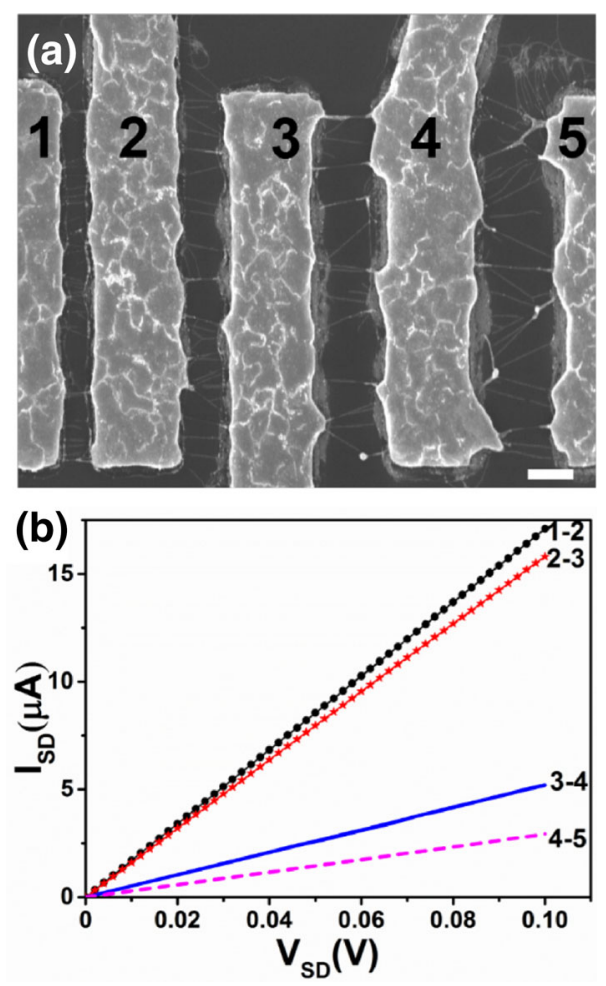

Fig. 1 a SEM micrograph of SWNTs between five different electrodes labeled as 1, 2, 3, 4 and 5. The designed spacing between the electrodes $1-2,2-3,3-4$ and $4-5$ is $1.0,2.0,3.0$ and $4.0 \mu \mathrm{m}$, respectively. The alignment of SWNTs depends on the spacing and the smallest spacing has the best alignment. Scale bar $2 \mu \mathrm{m}$. b Source-drain current $\left(I_{\mathrm{SD}}\right)$ vs. source-drain voltage $\left(V_{\mathrm{SD}}\right)$ of SWNTs. The corresponding resistance is $5.8,6.2,19.1$ and $34.2 \mathrm{k} \Omega$ between electrodes $1-2,2-3,3-4$ and $4-5$, respectively 
devices. Linear current-voltage curves with low electrical resistances of $5.8,6.2,19.1$ and $34.2 \mathrm{k} \Omega$, for electrodes 1-2, 2-3, 3-4 and 4-5, respectively, imply ohmic contact between SWNTs and electrodes for the fabricated devices. In addition, an increase in the resistance is observed as the spacing between electrodes increases. One possible explanation is that for carbon nanotubes whose length is greater than its mean-free path [the mean-free path of electrons in a CNT is typically $\sim 1 \mu \mathrm{m}$ (Park et al. 2004)], the resistance increases with the length, as shown in the equation below (Ando and Suzuura 2002; Datta 1995):

$R_{\mathrm{CNT}}=\left(\frac{h}{4 e^{2}}\right) \frac{L}{L_{\mathrm{o}}}$,

where $h$ is the Planck's constant, $e$ is the electron charge, $L_{\mathrm{o}}$ is the mean-free path and $L$ is the length of the CNT.

The SEM image shown in Fig. 2a was taken with high magnification and is the same as in Fig. 1a between electrodes 4-5. The SWNTs in the blue and red areas were characterized with AFM in tapping mode and the corresponding AFM images are shown in Fig. 2b (blue-dashed box) and c (red-dashed box). It is noted that while some SWNTs are detected in AFM topography, others are hardly
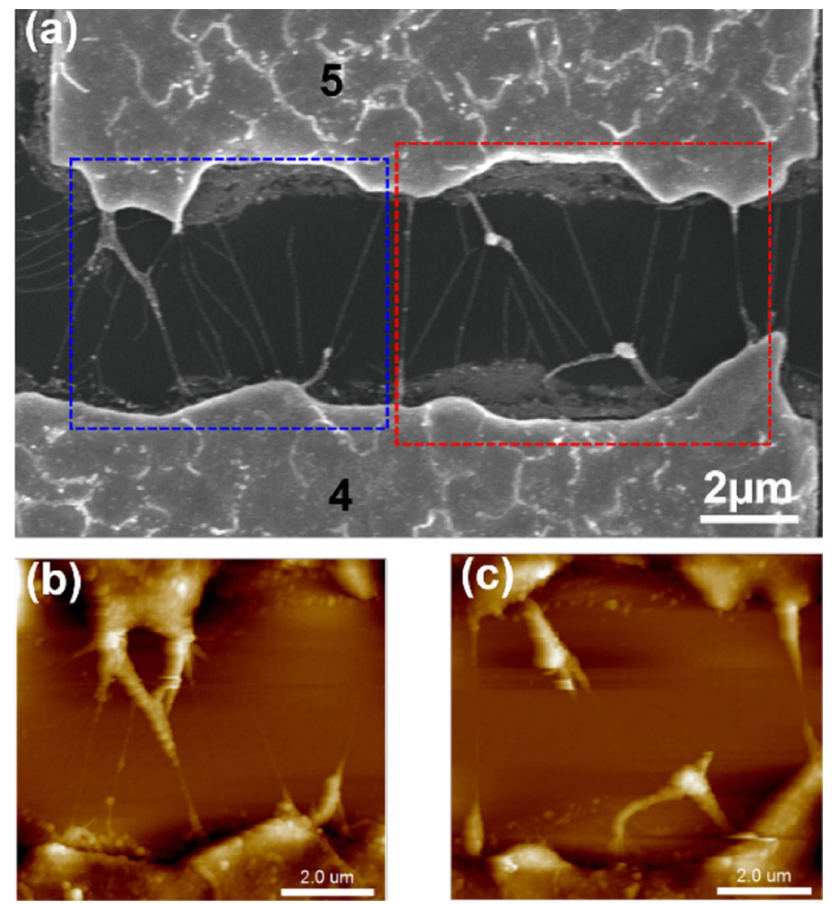

Fig. 2 a SEM image with high magnification. This image is same as that shown in Fig. 1a between electrodes 4-5. The SWNTs in the areas marked with blue- and red-dashed boxes are characterized with AFM, which is used to show if the SWNTs are suspended or lying on the substrate. b, c Corresponding AFM topographic images of the areas indicated in a by blue- and red-dashed boxes, respectively. The suspended SWNTs could be detected by AFM, while those lying on the substrate are not visible due to large height difference. AFM $z$ range $z=600 \mathrm{~nm}$ seen or not visible at all, which is due to the significant difference in height between the nanotubes and the electrodes. This implies that in our sample, some SWNTs are suspended and others are lying on the substrate, with the suspended SWNTs being visible in AFM topography. In addition, as revealed by the topography, the diameter of the suspended nanotubes appears larger than their actual sizes due to the curvature radius of the tip apex (approximately $20 \mathrm{~nm}$ ) and the spatial fluctuation of the suspended SWNTs caused by the intermittent contact with the tip (Ono and Ogino 2009).

The main purpose of our experiment is to control the number of SWNT channels between the two electrodes through manipulation of the device. AFM as a powerful tool not only for imaging nanoscale objects but also for the development of nanoscale electronic devices, has been used to perform the manipulation. In Fig. 3, we show SEM images obtained after several intermediate steps of our manipulation experiment. The initial configuration of SWNTs is shown in Fig. 3a. The SEM image shown in Fig. 3b was obtained after the first scanning with AFM in tapping mode. The structure of most nanotubes remained unchanged which suggests their high mechanical strength and a good contact stability between the nanotubes and the electrodes. Other nanotubes, indicated by white arrows, were either bent (right arrow) or cut and dissociated from the bundles. Their interaction within the bundle was overcome by the normal force applied by the tip (Ziyong et al. 2003).

To increase the interaction between the tip and the SWNTs, contact mode was used, where the tip and the nanotubes are in close contact, with almost no tunneling barrier between them. Efficient scanning parameters were established such as setpoint and tip bias. In contact mode, the setpoint refers to the deflection of the cantilever, which is directly proportional to the force exerted by the cantilever to the sample via Hooke's Law. Therefore, the tip force load can be controlled by adjusting the deflection setpoint of the tip. To obtain a more effective interaction time between the tip and the nanotubes, a slow scan speed was chosen which was fixed at $2 \mu \mathrm{m} / \mathrm{s}$ for all manipulation experiments. By gradually increasing the tip deflection setpoint, the tip force load was increased. Figure $3 \mathrm{c}$ shows SEM image obtained after the second AFM scanning using contact mode. The tip was placed in the area marked by a white rectangular box. By adjusting the tip force load to around $58 \mathrm{nN}$ with no bias applied to the tip, the nanotube bundles were disentangled into fluffy individual tubes, instead of translating or cutting. As a matter of fact, the manipulation of SWNTs is not only dependent on the interaction between the tip and the SWNTs but also on the binding forces between SWNTs within the bundle as well as the interaction between the SWNTs and the substrate (Claes and Larson 2001; Hertel et al. 1998; Ziyong et al. 

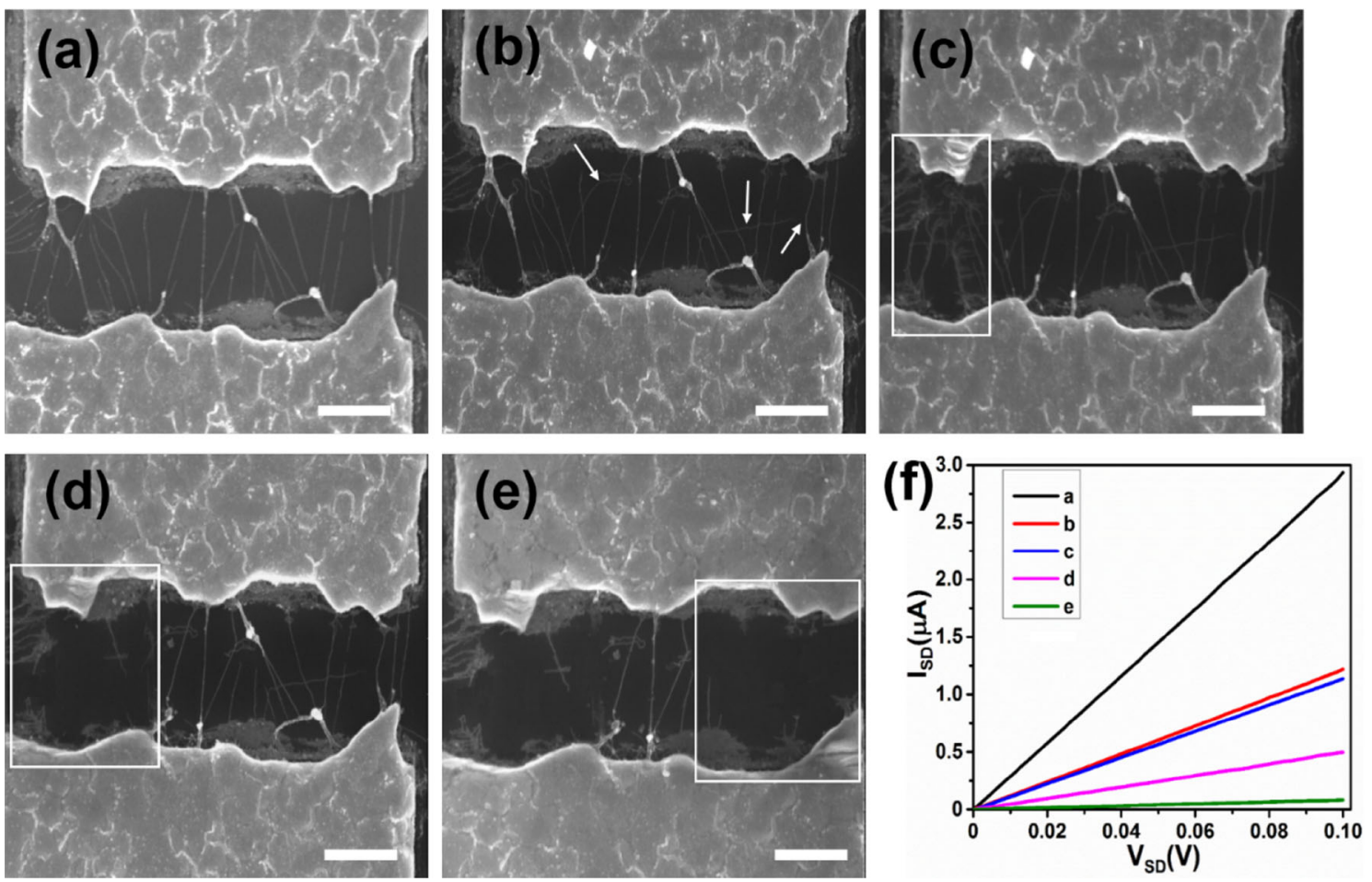

Fig. 3 a-e A series of SEM images showing the SWNTs after AFM manipulation. a The initial configuration of SWNTs. b SEM image of the SWNTs after the 1st scanning with AFM in tapping mode. Some SWNTs indicated by white arrows were either bent (right arrow) or cut by the tip and dissociated from the bundle. c SEM image of the SWNTs after the 2nd scanning with AFM in contact mode in the area marked by a white rectangle. By adjusting the tip-sample force to around $58 \mathrm{nN}$ at a scan speed of $2 \mu \mathrm{m} / \mathrm{s}$ and with no tip bias, the nanotube bundles were disentangled into fluffy individual tubes. d SEM image of the SWNTs after contact mode AFM scanning of the

2003). Hence, under mechanical forces and depending on the magnitude of the tip force applied, some deformations are likely to happen to the SWNTs such as rolling, sliding, bending, distortion and in our case, disentanglement.

Elsewhere, carbon nanotubes were reported to be cut by a biased AFM tip. Therefore, to cut or sweep away the nanotubes, we applied a bias to the AFM tip. A negative bias of $-10 \mathrm{~V}$ was shown to cut the SWNTs (not shown), consistent with recent experimental reports of cutting CNTs with a negatively biased tip (Hyon et al. 2005; Kim et al. 2003; Park et al. 2002). Multiple scans over the area marked with a white rectangular box in Fig. 3d resulted in SWNTs being completely swept away and picked up by the negatively charged tip. Figure $3 \mathrm{e}$ also shows an SEM image of the SWNTs after scanning with AFM contact mode in the area marked with a white rectangle. The area was scanned several times at a tip bias of $-10 \mathrm{~V}$ to completely remove all SWNTs. It can be seen that only three SWNTs remain bridging the electrodes.

Electrical characteristics were measured after every manipulation step. Figure $3 f$ shows room-temperature area marked with a white rectangle. By applying a negative tip bias of $-10 \mathrm{~V}$, the SWNTs were cut and swept away by the tip. The scanning was repeated several times until there were no SWNTs found. e SEM image of the SWNTs after contact mode AFM scanning of the area marked with a white rectangle. The area was scanned several times to remove all SWNTs. It can be seen that only three SWNTs remain bridging the electrodes. Scale bar $2 \mu \mathrm{m}$. f Source-drain current $\left(I_{\mathrm{SD}}\right)$ vs. source-drain voltage $\left(V_{\mathrm{SD}}\right)$ of SWNTs. The resistance of the initial configuration was $34.2 \mathrm{k} \Omega$ and increased to 82.1, 85.2, 202.4 and $1188.9 \mathrm{k} \Omega$ after each manipulation step, respectively

source-drain $I-V$ curves measured after every stage of manipulation. The resistance of the initial configuration was $34.2 \mathrm{k} \Omega$ and increased to $82.1 \mathrm{k} \Omega$ after AFM scanning with tapping mode in Fig. 3b. From the SEM result in Fig. 3a and $b$, we count around 18 nanotubes bridging the electrodes. After the manipulation in contact mode with no tip bias in Fig. 3c, the resistance increased slightly to $85.2 \mathrm{k} \Omega$, while it increased dramatically to $202.4 \mathrm{k} \Omega$ with the application of the negative bias to the tip (Fig. 3d). After this step, around 11 nanotubes are counted remaining between the electrodes. The manipulation in Fig. 3e resulted in a device with only three SWNTs between the electrodes and the resistance became larger to one order of magnitude, around $1188.9 \mathrm{k} \Omega$. Obviously, there is no linear relationship between the number of SWNTs and the change in resistance probably because our SWNT sample contains a mixture of both semiconducting and metallic SWNTs. Selective fabrication of highly purified SWNTs with controlled chirality has always been a challenge (Chen et al. 2013). With the advances in producing arrays of SWNTs with just one type of nanotube, our manipulation technique 
could be useful for controlling the channels between electrodes and hence investigating different SWNT devices with adjustable electronic properties.

\section{Conclusions}

In summary, we have reported the use of AFM to manipulate and control the conducting channels of SWNT arrays between a pair of palladium electrodes. Our results show that the tip-sample force as high as $58 \mathrm{nN}$ is necessary to disentangle the SWNT bundles and the application of a negative tip bias as high as $-10 \mathrm{~V}$ will always cut and sweep away the nanotubes. A final device with only three conducting SWNT channels between the electrodes was fabricated with a resistance of one order of magnitude larger than the initial device. This manipulation method provides the possibility to controllably modify the electrical properties of CNT-based devices for future electronic applications.

Acknowledgements This work was supported by National Science Foundation of China (Grant No. 51472057) and the Major Nanoprojects of Ministry of Science and Technology of China (2016YFA0200403). The first author acknowledges the financial support from CAS-TWAS President's fellowship program.

Open Access This article is distributed under the terms of the Creative Commons Attribution 4.0 International License (http:// creativecommons.org/licenses/by/4.0/), which permits unrestricted use, distribution, and reproduction in any medium, provided you give appropriate credit to the original author(s) and the source, provide a link to the Creative Commons license, and indicate if changes were made.

\section{References}

Ando T, Suzuura H (2002) Presence of perfectly conducting channel in metallic carbon nanotubes. J Phys Soc Jpn 71:2753-2760. doi:10.1143/jpsj.71.2753

Avouris P, Hertel T, Martel R, Schmidt T, Shea HR, Walkup RE (1999) Carbon nanotubes: nanomechanics, manipulation, and electronic devices. Appl Surf Sci 141:201-209. doi:10.1016/ S0169-4332(98)00506-6

Baughman RH, Zakhidov AA, de Heer WA (2002) Carbon nanotubes-the route toward applications. Science 297:787-792. doi:10.1126/science. 1060928

Chen Y et al (2013) Helicity-dependent single-walled carbon nanotube alignment on graphite for helical angle and handedness recognition. Nat Comm 4:2205. doi:10.1038/ncomms3205

Claes T, Larson S (2001) AFM manipulation of carbon nanotubes: realization of ultra-fine nanoelectrodes. Nanotechnology 13:108-113. doi:10.1088/0957-4484/13/1/323

Datta S (1995) Conductance from transmission in electronic transport in mesoscopic systems. Cambridge University Press, Cambridge, pp 48-116. doi:10.1017/CBO9780511805776.003

De Volder MFL, Tawfick SH, Baughman RH, Hart AJ (2013) Carbon nanotubes: present and future commercial applications. Science 339:535-539. doi:10.1126/science. 1222453
Fischer JE, Johnson AT (1999) Electronic properties of carbon nanotubes. Curr Opin Solid State Mat Sci 4:28-33. doi:10.1016/ s1359-0286(99)80007-2

Hertel T, Martel R, Avouris P (1998) Manipulation of individual carbon nanotubes and their interaction with surfaces. J Phys Chem B 102:910-915. doi:10.1021/jp9734686

Hyon CK, Atsuhiko K, Takafumi K, Masatoshi M, Kazuhiko M (2005) Non contact atomic force microscope electrical manipulation of carbon nanotubes and its application to fabrication of a room temperature operating single electron transistor. Jpn J Appl Phys 44:2056. doi:10.1143/jjap.44.2056

Ionescu AM, Riel H (2011) Tunnel field-effect transistors as energyefficient electronic switches. Nature 479:329-337. doi:10.1038/ nature 10679

Jiao L, Xian X, Fan B, Wu Z, Zhang J, Liu Z (2008) Fabrication of carbon nanotube diode with atomic force microscopy manipulation. J Phys Chem C 112:7544-7546. doi:10.1021/jp8020744

Karimov KS, Sulaiman K, Ahmad Z, Akhmedov KM, Mateen A (2015) Novel pressure and displacement sensors based on carbon nanotubes. Chin Phys B. doi:10.1088/1674-1056/24/1/018801

Kauffman DR, Star A (2008) Carbon nanotube gas and vapor sensors. Angew Chem Int Ed 47:6550-6570. doi:10.1002/anie.200704488

Kim DH, Koo JY, Kim JJ (2003) Cutting of multiwalled carbon nanotubes by a negative voltage tip of an atomic force microscope: a possible mechanism. Phys Rev B 68:113406. doi:10.1103/PhysRevB.68.113406

Kumar S, Kaur I, Kumari N, Jain S, Dharamveer K, Jindal VK, Verma NK, Bharadwaj LM (2012) Atomic force microscope manipulation of multiwalled and single walled carbon nanotubes with reflux and ultrasonic treatments. Appl Nanosci 4:19-26. doi:10.1007/s13204-012-0166-9

Li J, Yue W, Guo Z, Yang Y, Wang X, Syed AA, Zhang Y (2014) Unique characteristics of vertical carbon nanotube field-effect transistors on silicon. Nano Micro Lett 6:287-292. doi:10.1007/ bf03353793

Louie SG (2001) Electronic properties, junctions, and defects of carbon nanotubes. Top Appl Phys 80:113-145

Ono Y, Ogino T (2009) Observation of suspended carbon nanotube configurations using an atomic force microscopy tip. Jpn J Appl Phys 48:081601. doi:10.1143/jjap.48.081601

Park J, Yaish Y, Brink M, Rosenblatt S, McEuen PL (2002) Electrical cutting and nicking of carbon nanotubes using an atomic force microscope. Appl Phys Lett 80:4446-4448. doi:10.1063/1.1485126

Park J, Rosenblatt S, Yaish Y, Sazonova V, Ustunel H, Braig S, Arias TA, Brouwer PW, McEuen PL (2004) Electron-phonon scattering in metallic single-walled carbon nanotubes. Nano Lett 4:517-520. doi:10.1021/n1035258c

Postma HWC, Sellmeijer A, Dekker C (2000) Manipulation and imaging of individual single-walled carbon nanotubes with an atomic force microscope. Adv Mat 12:1299-1302. doi:10.1002/ 1521-4095(200009)12:17<1299:AID-ADMA1299>3.0.CO;2-O

Postma HWC, Teepen T, Yao Z, Grifoni M, Dekker C (2001) Carbon nanotube single-electron transistors at room temperature. Science 293:76-79. doi:10.1126/science.1061797

Salvetat JP, Bonard JM, Thomson NH, Kulik AJ, Forro L, Benoit W, Zuppiroli L (1999) Mechanical properties of carbon nanotubes. Appl Phys A 69:255-260. doi:10.1007/s003390050999

Seidel R, Graham AP, Unger E, Duesberg GS, Liebau M, Steinhoegl W, Kreupl F, Hoenlein W, Pompe W (2004) High-current nanotube transistors. Nano Lett 4:831-834. doi:10.1021/n1049776e

Zhang M (2013) Improving the electrical contact property of singlewalled carbon nanotube arrays by electrodeposition. Nano Micro Lett 5:242-246. doi:10.1007/bf03353755

Zhao Y et al (2008) Individual water-filled single-walled carbon nanotubes as hydroelectric power converters. Adv Mat 20:1772-1776. doi:10.1002/adma.200702956

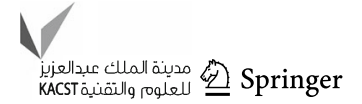


Ziyong S, Saijin L, Shimin H, Zhennan G, Zengquan X (2003) In situ splitting of carbon nanotube bundles with atomic force microscopy. J Phys D Appl Phys 36:2050. doi:10.1088/0022$3727 / 36 / 17 / 305$

\section{Publisher's Note}

Springer Nature remains neutral with regard to jurisdictional claims in published maps and institutional affiliations. 